\title{
Trauernde Angehörige - Wenn der Tierarzt zum Psychotherapeuten wird
}

\author{
Karolina Kantor
}

\begin{abstract}
Der Verlust eines Tieres ist für die meisten Tierhalter eine große psychische Belastung. Wie kann der Tierarzt in dieser schwierigen Zeit unterstützen? Dies möchte ich Ihnen aus zwei verschiedenen Blickrichtungen erläutern - einerseits als Fachärztin für Psychiatrie, andererseits als betroffene Tierhalterin, die innerhalb von einem Jahr zwei ihrer „Katzenkinder“ verlor.
\end{abstract}

\section{Was versteht man unter Trauer?}

Zunächst vielleicht zur Theorie: Eine Trauer nach Verlust einer nahestehenden Person - oder in diesem Fall eines Tieres - ist eine natürliche Reaktion, ohne dass zwangsläufig eine Erkrankung dahinterstehen muss. Sie besteht allerdings nicht nur aus Traurigkeit. In der Psychologie existieren mehrere Trauermodelle, u. a. das bekannteste von Kübler-Ross. Nach Bowlby (1961) und Parkes (1972) unterscheidet man 4 Trauerphasen:

- Phase I: Erschütterung - „Nicht-wahrhaben wollen“: vorwiegend mit einem Gefühl der emotionalen Betäubung bzw. Abstumpfung

- Phase II: Sehnsucht - intensives Verlangen und Suchen: In dieser Phase wird versucht, die Endgültigkeit des Todes zu leugnen. Diese Phase geht mit z. T. sehr intensiven, schmerzhaften Gefühlen einher, wie z.B. Wut, Einsamkeit, tiefe Traurigkeit. Auch Schuldgefühle spielen eine Rolle.

- Phase III: Desorganisation - Unordnung und Verzweiflung: In dieser Phase kämpft der Betroffene mit größeren oder kleineren Schwierigkeiten, seinen normalen Alltag zu meistern.

- Phase IV: Neuorientierung: Es ist die Zeit, in der der Betroffene versucht, den Tod in seinen Alltag zu integrieren und zurück ins normale Leben zu kommen.

Es ist jedoch keine streng vorbeschriebene Reihenfolge, die Phasen wechseln oft schnell untereinander und nicht jeder Betroffene muss alle 4 Phasen in der gleichen Ausprägung durchleben. Musikalisch wird diese Komplexizität der Trauer sehr bildhaft in „Marche funèbre“, dem 3. Satz der Klaviersonate b-Moll op. 35 von Fryderyk Chopin dargestellt. Und da jede Trauerreaktion sehr individuell ist, gibt es keine einzelne Wundermethode, wie man den Betroffenen unterstützen kann.
Was kann denn aber der Tierarzt tun, um den Tierbesitzer durch diese schwierige Zeit zu begleiten - ohne dabei die Therapeutenrolle annehmen zu müssen?

\section{Aufklärung über die infauste Prognose}

Eine adäquate Aufklärung ist ein wichtiger Punkt jeder guten Behandlung. Sie muss nicht immer sehr ausführlich und detailliert sein, viel wichtiger ist, dass sie verständ-

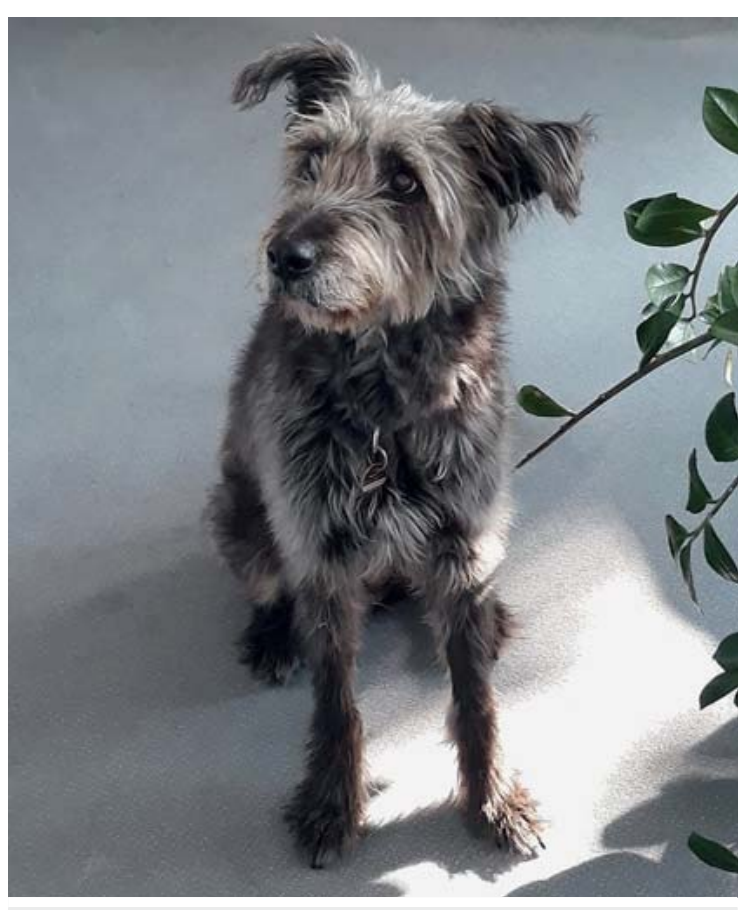

- Abb. 1 In den meisten Fällen ist es nicht „nur ein Tier“, sondern ein Familienmitglied, von dem sich der Tierhalter verabschieden muss. Quelle: K. Kalenyak 


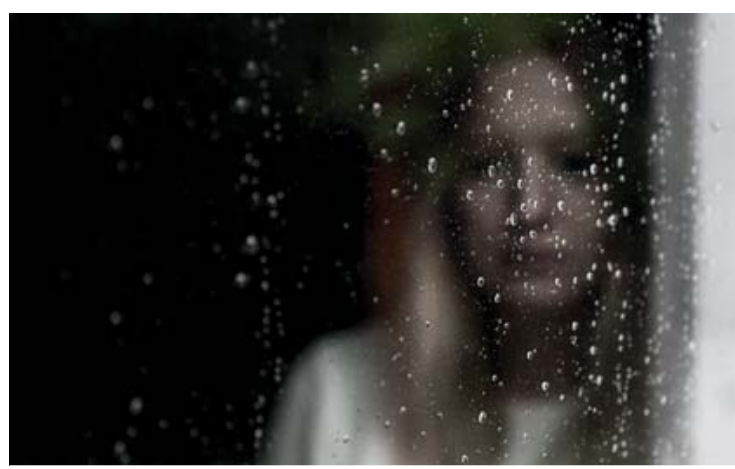

Abb. 2 Trauern gehört dazu. Sollte ein Tierhalter jedoch noch Wochen nach einem Verlust deprimiert oder gereizt wirken, sollten Sie sich um seine psychische Gesundheit Sorgen machen. Quelle: Kirsten Oborny/Thieme Gruppe

lich ist. Je nachdem, was für einen Menschen man vor sich hat, sollte man entscheiden, welche Wortwahl man nimmt und wie viele medizinische Einzelheiten man anspricht. Auch wenn es am Anfang sehr zeitaufwendig und schwer in den Praxisalltag integrierbar erscheint, lohnt es sich häufig, 10 Minuten mehr in das Aufklärungsgespräch zu investieren und Raum für Fragen zu lassen. Oft sind nämlich die Ungewissheit und das unzureichende Verständnis für die Situation der Grund für die - nicht selten emotional geladenen - Reaktionen der Tierhalter, die auch Tage und Wochen nach dem Tod oder der Euthanasie des Tieres vorkommen.

Von besonderem Wert sind die Ursachen der Erkrankung sowie die Therapieoptionen, denn hier treten am häufigsten Zweifel auf. Die Lage sollte kritisch und gleichzeitig aber auch offen und ehrlich besprochen werden. Soweit die medizinische Situation es zulässt, sollte man den Angehörigen Zeit lassen, bevor sie ihre Entscheidung treffen. Meiner Meinung nach sind Ehrlichkeit und Transparenz besonders wichtig - auch wenn die Prognose infaust ist.

Solche Botschaften können natürlich sehr emotional empfangen werden, im Nachhinein ist man dann aber doch dankbar, wenn in so einer belastenden Situation keine falsche Hoffnung gegeben wird. Gleichzeitig ist es aber wichtig, dem Tierhalter keine inadäquate Angst zu machen oder (direkt bzw. indirekt) Druck aufzubauen. Ich persönlich war sehr entlastet, als mich nach fast einer Woche stationärer Diagnostik bei meinem geliebten Kater die Hämatologin, die wir konsultiert haben, anrief, um mir mitzuteilen, dass es sich um eine schwere und aggressive Form der AML handelt, die nicht mehr heilbar ist. Es war kein angenehmes Telefonat, ich konnte aber immer darauf zurückgreifen, als mich das schlechte Gewissen überkam.

Im Falle einer Euthanasie ist es wichtig, den Angehörigen den Ablauf oder zumindest das Prinzip dieses Vorgangs zu erklären, um ihnen die unnötige Angst (z. B. dass das Tier währenddessen leiden muss) zu nehmen.

\section{Mein Tier ist gestorben - wie geht das Leben weiter?}

Egal wie gut man aufgeklärt und vorbereitet wird, ist der Tod des Tieres oft ein sehr belastendes Erlebnis. Soweit es möglich ist, sollte man den Angehörigen die Möglichkeit geben, sich von dem verstorbenen Tier zu verabschieden und kurz in einem gesonderten Raum den ersten Schock zu überstehen. Nicht jeder möchte unmittelbar danach darüber reden, oft fallen einem die Fragen erst Stunden, Tage oder Wochen danach ein. Trotzdem ist es wichtig, den Betroffenen ernst zu nehmen und ihm zu vermitteln, dass seine Trauer völlig angemessen ist.

Als Tierhalter hört man in dieser Situation oft von seiner Umgebung „Hab dich nicht so, es war doch nur ein Tier, hol dir einfach ein neues“. Dies führt dazu, dass sich der Betroffene mit seiner Trauer unwohl fühlt und das Gefühl bekommt, überzureagieren. Hier kann ein Gespräch mit dem Tierarzt enorm helfen, insbesondere wenn Sie den Betroffenen bereits einigermaßen gut kennen. Zeigen Sie Wertschätzung und Verständnis, sagen Sie dem Tierhalter, dass er nicht der einzige ist, der über sein verstorbenes Tier trauert und dass seine Gefühle völlig adäquat sind. Wenn Ihnen eine ähnliche Geschichte einfällt, nutzen Sie sie als Beispiel - auch wenn es sich etwas trivial anhört, hilft es in den schwierigen Zeiten zu hören, dass es den anderen auch so geht.

Animieren Sie den Angehörigen, sich mit anderen Betroffenen in Verbindung zu setzen - sei es im Rahmen einer Selbsthilfegruppe oder eines Internetforums. Wenn der Tierhalter sich nicht in der Lage fühlt, seiner beruflichen Tätigkeit nachzugehen, empfehlen Sie ihm eine Vorstellung beim Hausarzt zwecks einer kurzzeitigen Krankschreibung - viele machen es nicht aus Scham und Angst, sich lächerlich zu machen; „es war doch nur ein Tier“.

\section{Wie kann der Tierarzt in der Trauerzeit helfen?}

Als Tierarzt ist man in den hier beschriebenen Trauerfällen einer der wichtigsten Begleiter des Betroffenen. Achten Sie jedoch dabei auf Ihre persönlichen Grenzen und geben Sie nur so viel, dass es auch für Sie erträglich ist.

Im Folgenden habe ich einige Tipps gesammelt:

- Versuchen Sie, der Kommunikation eine Struktur zu setzen. Vergeben Sie Termine für die Angehörigengespräche - und zwar nicht mit der Botschaft „Ich habe jetzt keine Zeit“, sondern „Ich nehme mir gerne für Sie Zeit“. Wenn Menschen unter starker Belastung stehen, sind sie oft etwas kritikloser als sonst - die meisten werden aber verstehen, dass Sie z. B. nicht jeden Tag außerhalb Ihrer Sprechzeiten oder mitten in 
der Sprechstunde mit ihnen telefonieren können und sie bleiben Ihnen trotzdem für die Zuwendung dankbar.

- Auch die eigenen körperlichen Grenzen sind wichtig - nicht jeder mag es, fremde Menschen zu umarmen oder „Händchen zu halten“, und wenn Sie auch nicht zu dieser Gruppe gehören, müssen Sie es nicht tun. Nähe und Unterstützung können genauso gut mit Worten und Mimik übermittelt werden.

- Versuchen Sie, weinenden Angehörigen eine Rückzugsmöglichkeit zu geben - sei es ein separater Raum oder der Innenhof der Praxis. Es vermittelt das Gefühl von Geborgenheit und Vertrauen, nicht nur für Menschen, die sich für ihre emotionale Reaktion schämen.

- Sie sind nicht verpflichtet, sich mit inadäquaten Äußerungen auseinander zu setzen. Jegliche Art von Bedrohungen oder Beleidigungen sollte nicht toleriert werden. In diesem Fall beenden Sie das Gespräch, da es nicht mehr zielführend ist und nur als Ventil für die kumulierten Emotionen dient.

- Achten Sie auf die eigene Psychohygiene, insbesondere wenn es um Fälle geht, die Sie selbst emotional belasten. Eine gute Möglichkeit ist ein imaginativer „Tresor“, den Sie z. B. vor der Praxistür platzieren können. Lassen Sie sich nach dem Feierabend kurz Zeit, um die Gefühle und Gedanken dort zu „verschließen“, um sie nicht mit nach Hause zu nehmen. Je nach persönlichen Eigenschaften braucht dieser Prozess mehr oder weniger Zeit, bis es richtig funktioniert, er ist aber sehr hilfreich.

- Tauschen Sie sich bei schwierigen Fällen mit Ihren Kollegen aus - vielleicht hatte jemand schon eine ähnliche Situation erlebt und Sie können aus seiner Erfahrung lernen, wie Sie damit umgehen können?

\section{Wann wird Trauer pathologisch?}

In dem neuen Klassifikationssystem der Krankheiten der WHO ICD-11, wird die prolongierte Trauerreaktion als eine separate Einheit kodiert. Davon spricht man, wenn die Reaktion länger als 6 Monate dauert und zu deutlichen Beeinträchtigungen des Alltags und der sozialen Kontakte des Betroffenen führt. Der Betroffene zeigt eine überdauernd starke Sehnsucht nach dem Verstorbenen, übermäßige Schuldgefühle, intensiv empfundene Emotionen wie Trauer oder Wut und verminderte Fähigkeit zu positiven Gefühlen.

Außerdem spricht man auch von einer abnormen Trauerreaktion, die nicht nur durch eine überdurchschnittliche Dauer, sondern auch eine Art und Intensität, die weit über die gesellschaftlichen und kulturellen Normen hinausreicht. Bestimmte Trauerphasen werden dabei nicht erlebt oder sie zeigen ein pathologisches Ausmaß. Es kann z.B. mit extremer Traurigkeit und Weinerlichkeit, starker Gereiztheit bis zur Aggressivität, massiven Schuldgefühlen usw. einhergehen.
Wenn der Betroffene nach längerer Zeit (über 4 Wochen) weiterhin stark deprimiert wirkt, im Kontakt sehr gereizt und aggressiv oder massiv auf die eigenen Schuldgefühle fixiert ist, sollte man sich um seine psychische Gesundheit Sorgen machen. In diesem Fall ist es wichtig, den Betroffenen zu einer Vorstellung, z. B. bei seinem Hausarzt, zu motivieren, da es sich um eine behandlungspflichtige depressive Erkrankung handeln könnte.

Auch die Äußerung von Suizidgedanken sollte auf jeden Fall ernst genommen werden. Wenn der Betroffene direkt droht, sich das Leben zu nehmen, sollte dringend medizinische Hilfe hinzugezogen werden. Bei schwerer und bedrohlicher Aggressivität muss ggf. die Polizei alarmiert werden, um die Gefahr für das Praxisteam und andere Patienten zu eliminieren.

\section{Muss also der Tierarzt zum Therapeuten werden?}

Zusammenfassend ist festzustellen, dass die Rolle des Tierarztes in der Trauerbewältigung sehr wichtig ist und sehr stark dazu beitragen kann, dass die Tierhalter die schwierige Zeit nach dem Verlust eines Tieres besser überwinden. Auf keinen Fall müssen Sie aber die Verantwortung für die psychische Gesundheit Ihrer „menschlichen“ Klienten übernehmen und sich Vorwürfe machen, wenn es zu einer der oben beschriebenen Situationen kommen sollte.

Seien Sie empathisch, schenken Sie den Angehörigen ein bisschen von Ihrer Zeit und Aufmerksamkeit - damit ist im Trauerfall schon enorm geholfen. Und ganz wichtig vergessen Sie dabei nicht die Selbstfürsorge!

\section{Korrespondenzadresse}

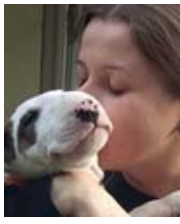

\section{Karolina Kantor}

Fachärztin für Psychiatrie und Psychotherapie Universitätsklinikum Halle (Saale) Universitätsklinik und Poliklinik für Psychiatrie, Psychotherapie und Psychosomatik Julius-Kühn-Str. 7, 06112 Halle/Saale karolina.kantor@uk-halle.de

Literatur

[1] Vorderholzer U, Hohagen F. Therapie psychischer Erkrankungen. State oft the art. München: Elsevier Urban \& Fischer; 2018

[2] Lieb K, Frauenknecht S. Intensivkurs Psychiatrie und Psychotherapie. München: Elsevier Urban \& Fischer; 2019

\section{Bibliografie}

DOI https://doi.org/10.1055/a-1208-0951

Kleintier konkret 2020; 23: 44-46

(c) Georg Thieme Verlag KG Stuttgart · New York ISSN 1434-9132 